\title{
A Uniformly Convergent Finite Difference Scheme for a Singularly Perturbed Semilinear Equation
}

Paul A. Farrell, John J. H. Miller, Eugene O'Riordan, and Grigori I. Shishkin

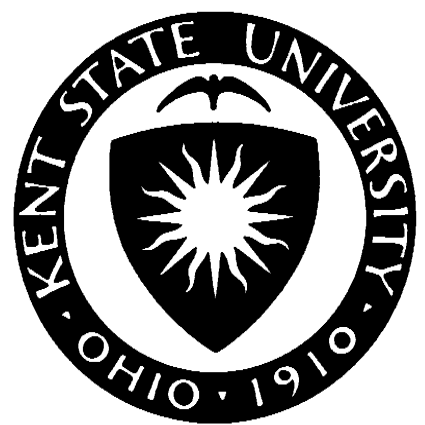

\section{Computer Science Program}

Technical Report Number CS-9405-14

August 31, 1995

Department of Mathematics and Computer Science

Kent State University

Kent, Ohio 44242. 


\title{
A UNIFORMLY CONVERGENT FINITE DIFFERENCE SCHEME FOR A SINGULARLY PERTURBED SEMILINEAR EQUATION
}

\author{
PAUL A. FARRELL *, JOHN J. H. MILLER ${ }^{\dagger}$, EUGENE O’RIORDAN ${ }^{\dagger}$ AND GRIGORI I. \\ SHISHKIN §
}

\begin{abstract}
Boundary value problems for singularly perturbed semilinear elliptic equations are considered. Special piecewise-uniform meshes are constructed which yield accurate numerical solutions irrespective of the value of the small parameter. Numerical methods composed of standard monotone finite difference operators and these piecewise-uniform meshes are shown theoretically to be uniformly (with respect to the singular perturbation parameter) convergent. Numerical results are also presented, which indicate that in practice the method is first order accurate.
\end{abstract}

AMS(MOS) subject classifications. 34E10, 65L10.

Key words. Semilinear boundary value problem, singular perturbation, finite-difference scheme, piecewise uniform mesh.

1. Introduction. Partial differential equations with a small parameter $\varepsilon$ multiplying the highest order derivative terms are said to be singularly perturbed. Singularly perturbed differential equations are all pervasive in applications of mathematics to problmes in the sciences and engineering. Among these are the Navier-Stokes equations of fluid flow at high Reynolds number, the drift-diffusion equations of semiconductor device physics [17, 12], the Michaelis-Menten theory for enzyme reactions [16], and mathematical models of liquid crystal materials and of chemical reactions [25].

The use of classical numerical methods for solving such problems may give rise to difficulties when the singular perturbation parameter $\varepsilon$ is small. In particular, methods based on centered differences or upwinded differences on uniform meshes yield error bounds, in the maximum norm, which depend on an inverse power of $\varepsilon$. Similarly Brandt and Yavneh [1] demonstrated that anisotropic artificial viscosity in the first-order upwind finitedifference scheme may result in inaccurate solutions, when $\varepsilon / h=O(1)$, where $h$ is the mesh width. Two alternative approaches may be taken to the resolution of this problem. Either additional information about the solution may be used to produce accurate efficient methods, which may involve a priori modification of the mesh or operator, or an attempt may be made to produce a postiori adaptive methods or black box methods.

The latter approach leads to codes that are designed to handle a wider variety of problems than non-adaptive codes, usually at the expense of greater execution time. Moreover, such methods are less suitable than non-adaptive codes to implementation in a parallel environment. This is because the adaption process inherent in a posteriori methods, introduces sequentiality to the solution process, which is absent in the a priori case. The

\footnotetext{
To appear in SIAM Journal of Numerical Analysis

* Department of Mathematics and Computer Science, Kent State University, Kent, Ohio 44242, U.S.A. The work of this author was supported in part by The Research Council of Kent State University

$\dagger$ Department of Mathematics, Trinity College, Dublin 2, Ireland

$\ddagger$ School of Mathematical Sciences, Dublin City University, Glasnevin, Dublin 9, Ireland

$\S$ Institute of Mathematics and Mechanics, Russian Academy of Sciences, Ekaterinburg, Russia.
} 
former approach uses physical or mathematical knowledge about the problem to enhance the solution strategy. Such methods are widespread in the literature. These include fitted finite difference methods, finite element methods using special elements such as exponential elements, and methods which use a priori refined or special meshes. Examples of these include methods for convection-diffusion problems devised by the British Central Electricity Generating Board [11], fluid flow in aerodynamics [3], semiconductor device physics $[18,4,15,14]$ and chemical reactions [24].

It is of theoretical and practical interest to consider numerical methods for such problems, which exhibit $\varepsilon$-uniform convergence, that is, numerical methods for which there exists an $N_{0}$, independent of $\varepsilon$, such that for all $N \geq N_{0}$, where $N$ is the number of mesh elements, the error constant and rate of convergence in the maximum norm are independent of $\varepsilon$. Thus a numerical method is said to be $\varepsilon$-uniform of order $p$ on the mesh $\Omega_{N}=\left\{x_{i}, i=0,1, \ldots, N\right\}$ if there exists an $N_{0}$ independent of $\varepsilon$ such that for all $N \geq N_{0}$

$$
\sup _{0<\varepsilon \leq 1} \max _{\Omega_{N}}\left|u(x)-u_{N}(x)\right| \leq C N^{-p},
$$

where $u$ is the solution of the differential equation, $u_{N}$ is the numerical approximation to $u, C$ and $p>0$ are independent of $\varepsilon$ and $N$.

Singularly perturbed boundary value problems for linear elliptic equations, which reduce for $\varepsilon=0$ to zero-order equations, were examined in $[10,19,20,21,22]$. For such problems $\varepsilon$-uniform methods consisting of exponentially fitted finite difference operators on uniform meshes were thoroughly investigated and applied successfully to ordinary differential equations in $[2,8]$ and to linear partial differential equations in $[10,19,20,21]$.

In $[6,13]$ it is shown that it is impossible to find an exponentially fitted finite difference operator on a uniform rectangular mesh which yields an $\varepsilon$-uniform numerical method for a certain class of one-dimensional semilinear differential equations. In this paper $\varepsilon$-uniform numerical methods are constructed for a class of semilinear problems, using classical finite difference operators on special piecewise-uniform meshes. Thus $\varepsilon$-uniform methods can be constructed on special piecewise uniform meshes even if it is not possible on uniform meshes. An added advantage of standard difference methods on a priori refined meshes over exponentially fitted methods arises from the non-linear nature of the difference schemes. These must be solved using an iterative technique. In the case of exponentially fitted methods, such as those in [2], assembly of the iteration matrix involves the evaluation of exponential or related hyperbolic functions in each iteration, since the fitting factor depends on the solution. Refined mesh methods such as those described here do not suffer from this disadvantage.

In $\S 2$ the continuous problem is formulated and bounds on the derivatives of the solution are given. In $\S 3$, a nonlinear finite difference method is constructed which is $\varepsilon$-uniform. In $\S 4$ this method is linearized by means of continuation, and it is shown that the resulting linear finite difference method is still $\varepsilon$-uniform. Finally, in $\$ 5$, numerical results are presented which support these theoretical results and show that in practice the methods are $\varepsilon$-uniform and are first order accurate.

2. Problem formulation. Consider the Dirichlet problem for the semilinear elliptic equation 


$$
\begin{gathered}
L u(x) \equiv \varepsilon^{2} L_{2} u(x)+\varepsilon L_{1} u(x)-g(x, u(x))=0, x \in \Omega, \\
u(x)=\phi(x), x \in \partial \Omega .
\end{gathered}
$$

on the $n$-dimensional infinite strip $\Omega=\left\{x \in \Re^{n}, 0<x_{1}<d\right\}$, where

$$
\begin{gathered}
L_{2} \equiv \sum_{r, s=1}^{n} a_{r s}(x) \frac{\partial^{2}}{\partial x_{r} \partial x_{s}}+\sum_{r=1}^{n} b_{r}(x) \frac{\partial}{\partial x_{r}}-c(x) \\
L_{1} \equiv \sum_{r=1}^{n} b_{r}^{1}(x) \frac{\partial}{\partial x_{r}}-c^{1}(x)
\end{gathered}
$$

are linear differential operators. The more general case, where the coefficients of $L_{1}$ and $L_{2}$ depend on $u(x)$, is dealt with in [13]. The coefficients $a_{r s}(x), b_{r}(x), c(x), b_{r}^{1}(x), c^{1}(x), r, s=$ $1, \ldots, n$, and the functions $g(x, u), \phi(x)$ are assumed to be sufficiently smooth on the sets $\bar{\Omega}, \bar{\Omega} \times \Re$ and $\partial \Omega$ respectively, and to satisfy the conditions

$$
\begin{gathered}
a_{0} \sum_{r=1}^{n} \xi_{r}^{2} \leq \sum_{r, s=1}^{n} a_{r s}(x) \xi_{r} \xi_{s} \leq a^{0} \sum_{r=1}^{n} \xi_{r}^{2}, x \in \Omega \\
a_{0}>0, c(x), c^{1}(x) \geq 0, x \in \bar{\Omega}
\end{gathered}
$$

The parameter $\varepsilon$ takes arbitrary values in the half-open interval $(0,1]$. In addition, it is assumed that the function $g(x, u)$ satisfies the condition

$$
\frac{\partial}{\partial u} g(x, u) \geq g_{0}>0, \quad(x, u) \in \bar{\Omega} \times\left[-M_{1}, M_{1}\right]
$$

where $M_{1}$ is a sufficiently large number. Throughout this paper, $M$ and $m$ (sometimes subscripted) denote positive constants that are independent of $\varepsilon$, and in the case of discrete problems also independent of the mesh. A function $u \in C^{2}(\Omega)$, continuous and bounded on $\bar{\Omega}$, is a solution of the boundary value problem (2.1) if it satisfies (2.1) on $\Omega$.

We shall find it useful later to use the formal notation,

$$
\mathcal{L}(f(x, w)) u(x)=\left(\varepsilon^{2} L_{2}+\varepsilon L_{1}\right) u(x)-f(x, w(x)) .
$$

In this notation the operator in $(2.1)$ is given by

$$
L u(x) \equiv \mathcal{L}(g(x, u)) u(x)
$$


Furthermore

$$
\mathcal{L}(g(x, v)) u(x) \equiv\left\{\varepsilon^{2} L_{2}+\varepsilon L_{1}\right\} u(x)-g(x, v(x)),
$$

where $v=v(x), v \in C^{1}(\Omega)$. The following lemma is proved in [9, Ch. 4, $\left.\S 8.1\right]$.

Lemma 2.1. Let $w_{i} \in C^{2}(\Omega) \cap C^{0}(\bar{\Omega})$ for $i=1,2$ and $x \in \bar{\Omega}$. Suppose that the inequalities

$$
\mathcal{L}(g(x, u)) w_{1}(x) \geq L u(x) \geq \mathcal{L}(g(x, u)) w_{2}(x)
$$

hold for all $x$ such that

$$
w_{1}(x)>u(x)>w_{2}(x) .
$$

Then, for all $x \in \bar{\Omega}$, the function $u$ satisfies the following inequalities

$$
\min \left\{w_{1}(x),-\operatorname{osc}\left[\left.w_{1}\right|_{\Omega}\right]+\min _{\partial \Omega} \phi\right\} \leq u(x) \leq \max \left\{w_{2}(x), \operatorname{osc}\left[\left.w_{2}\right|_{\Omega}\right]+\max _{\partial \Omega} \phi\right\},
$$

where $\operatorname{osc}\left[\left.v\right|_{\Omega}\right]$ is the oscillation of $v(x)$ on $\Omega$, that is the difference between essential $\max _{\Omega} v(x)$ and essential $\min _{\Omega} v(x)$.

Using this lemma it can be shown that

$$
|u(x)| \leq M_{2}, x \in \bar{\Omega},
$$

where

$$
M_{2}=\max \left[g_{0}^{-1} \max _{\bar{\Omega}}|g(x, 0)|, \max _{\partial \Omega}|\phi(x)|\right] .
$$

For the solution of a semilinear boundary value problem to be unique, it is necessary that, for each value of $\varepsilon, \max _{\bar{\Omega}}|u|$ and $\max _{\bar{\Omega}}|\nabla u|$ are bounded see [9, Ch. 4]. Since the operator containing second order derivatives can be written in divergence form, the estimate of $|\nabla u|$ depends only on $\max _{\bar{\Omega}}|u|, \varepsilon, a_{0}, a^{0}$ and on the distance to $\partial \Omega$. In a neighbourhood of $\partial \Omega$ it depends also on $|\phi|_{\Omega}^{(2)}[9$, Ch. 4, Theorem 4.1].

Assume that the data of Problem (2.1) satisfy

$$
\begin{gathered}
a_{r s}, b_{r}, c, b_{r}^{1}, c^{1} \in C^{\ell-1+\lambda}(\bar{\Omega}), g \in C^{\ell-1+\lambda}\left(\bar{\Omega} \times\left[-M_{2}, M_{2}\right]\right), \\
\phi \in C^{\ell+\lambda}(\partial \Omega), \quad r, s=1, \ldots, n, \ell \geq 2, \lambda>0
\end{gathered}
$$

Then $u \in C^{\ell+\lambda}(\bar{\Omega})$ (see $[9]$ ).

We are now in a position to state the following bounds on $u(x)$ and its derivatives:

Theorem 2.2. [13, Theorem 4.1, pg. 110] Suppose that the data of Problem (2.1) satisfy the bound (2.2) and the smoothness conditions (2.4). Then, for $\varepsilon \in(0,1]$,

$$
|u(x)| \leq M_{2}, x \in \bar{\Omega} \quad \text { and }
$$




$$
\begin{gathered}
\left|\frac{\partial^{|\alpha|}}{\partial x^{\alpha}} u(x)\right| \leq M \varepsilon^{-|\alpha|},|\alpha| \leq \ell, x \in \bar{\Omega},|\alpha|=\alpha_{1}+\ldots \alpha_{n}, \\
\frac{\partial^{|\alpha|}}{\partial x^{\alpha}} \equiv \frac{\partial^{|\alpha|}}{\partial x_{1}^{\alpha_{1}} \partial x_{2}^{\alpha_{2}} \ldots \partial x_{n}^{\alpha_{n}}}
\end{gathered}
$$

where $\alpha=\left(\alpha_{1}, \alpha_{2}, \ldots, \alpha_{n}\right)$ and $|\alpha|=\alpha_{1}+\alpha_{2}+\ldots+\alpha_{n}$.

In what follows, more precise information on the behaviour of the solution of $(2.1)$ is needed. To obtain this, the solution is written in the form

$$
u(x)=U(x)+V(x), x \in \bar{\Omega}
$$

where $U$ and $V$ are the regular and singular parts respectively of the solution $u$ of (2.1). Estimates of $U$ and $V$ are now derived separately.

The function $U$ is the solution of the equation $L(U(x))=0, x \in \Omega$ with boundary values $U_{0}$ on $\partial \Omega$. On $\bar{\Omega}, U_{0}$ is the solution of the equation $g\left(x, U_{0}(x)\right)=0$. Under the condition

$$
g \in C^{\ell_{1}}\left(\bar{\Omega} \times\left[-M_{3}, M_{3}\right]\right), \ell_{1} \geq 1
$$

where $M_{3}$ is a sufficiently large number, $U_{0}$ satisfies

$$
\left|\frac{\partial^{|\alpha|}}{\partial x^{\alpha}} U_{0}(x)\right| \leq M, \quad x \in \bar{\Omega},|\alpha| \leq \ell_{1} .
$$

On $\bar{\Omega}, U$ can be written as a sum of functions $U=U_{0}+v$, where $v$ is the solution of

$$
\mathcal{L}\left(\hat{g}\left(x, U, U_{0}\right)\right) v(x)=F_{1}\left(x, U_{0}\right), x \in \Omega, v(x)=0, x \in \partial \Omega
$$

where

$$
\hat{g}_{u}\left(x, v_{1}, v_{2}\right) \equiv \int_{0}^{1} \frac{\partial}{\partial u} g\left(x, \nu v_{1}+(1-\nu) v_{2}\right) d \nu
$$

and

$$
F_{1}(x, v) \equiv-\left\{\varepsilon^{2} L_{2}+\varepsilon L_{1}\right\} v(x)
$$

Under condition (2.7), it can be shown that $v$ satisfies

$$
\left|\frac{\partial^{|\alpha|}}{\partial x^{\alpha}} v(x)\right| \leq M \varepsilon^{1-|\alpha|}, x \in \bar{\Omega},|\alpha| \leq \ell_{1}-2
$$


which is similar to (2.5). From inequalities (2.8) and (2.10), the required bounds on the derivatives of $U$ are obtained:

$$
\left|\frac{\partial^{|\alpha|}}{\partial x^{\alpha}} U(x)\right| \leq M \varepsilon^{1-|\alpha|}, x \in \bar{\Omega},|\alpha| \leq \ell_{1}-2 .
$$

The function $\mathrm{V}$ is the solution of

$$
\mathcal{L}(\hat{g}(x, u, U)) V(x) x \in \Omega, V(x)=\phi(x)-U(x), x \in \partial \Omega .
$$

Let $m_{1}$ be a sufficiently small number satisfying

$$
g_{0}-a^{0}\left(m_{1}\right)^{2}-\max _{\bar{\Omega}}\left[\left|b_{1}(x)\right|+\left|b_{1}^{1}(x)\right|\right] m_{1} \geq m_{2} g_{0},
$$

where $m_{2} \in(0,1)$ is arbitrarily small. It follows from the maximum principle that

$$
|V(x)| \leq M \exp \left(-m_{3} \varepsilon^{-1} d(x)\right), x \in \bar{\Omega},
$$

where $m_{3} \leq m_{1}$ and $d(x)$ is the distance from $x$ to $\partial \Omega$.

With the change of variables $\xi=\xi(x), \xi_{r}=\varepsilon^{-1} x_{r}, r=1, \ldots, n,(2.14)$ leads to

$$
\left|\frac{\partial^{|\alpha|}}{\partial \xi^{\alpha}} \tilde{V}(\xi)\right| \leq M \exp \left(-m_{3} \tilde{d}(\xi)\right), \xi \in \overline{\tilde{\Omega}} .
$$

where $\tilde{d}(\xi)$ is the distance from $\xi$ to $\partial \tilde{\Omega}$, and $\tilde{\Omega}=\xi(\Omega)$ is a region of diameter $O(1 / \varepsilon)$. From this estimate the inequality

$$
\left|\frac{\partial^{|\alpha|}}{\partial x^{\alpha}} V(x)\right| \leq M \varepsilon^{-|\alpha|} \exp \left(-m_{3} \varepsilon^{-1} d(x)\right), x \in \bar{\Omega}
$$

follows.

The bounds on the derivatives $\left(\partial^{|\alpha|} / \partial x_{1}^{\alpha_{1}} \ldots \partial x_{n}^{\alpha_{n}}\right) V(x)$ for $\alpha_{1}<|\alpha|$ are now made more precise. Differentiating the equation in (2.12) with respect to $x_{r}, r=2, \ldots, n$, and putting $V^{(r)}=\left(\partial / \partial x_{r}\right) V$, it follows that

$$
\mathcal{L}\left(g_{u}(x, v)\right) V^{(r)}(x)=F_{2}(x, u, U, V), x \in \Omega,
$$

where $F_{2}$ comprises the terms of the derivative of $(2.12)$ not contained in $\mathcal{L}\left(g_{u}(x, v)\right) V^{(r)}(x)$. From (2.11) and (2.15) the inequality 


$$
\left|F_{2}(x, u, U, V)\right| \leq M \exp \left(-m_{3} \varepsilon^{-1} d(x)\right), x \in \bar{\Omega}
$$

is obtained. Furthermore, similarly to (2.15), the derivatives

$$
\left(\frac{\partial^{|\alpha|}}{\partial x^{\alpha}}\right) V^{(r)}(x)=\left(\frac{\partial^{|\alpha|+1}}{\partial x_{r} \partial x^{\alpha}}\right) V(x)
$$

satisfy

$$
\left|\frac{\partial^{|\alpha|}}{\partial x^{\alpha}} V^{(r)}(x)\right| \leq M\left[\varepsilon^{-\alpha_{1}}+\varepsilon^{1-|\alpha|}\right] \exp \left(-m_{3} \varepsilon^{-1} d(x)\right), x \in \bar{\Omega}
$$

Thus the following theorem holds

Theorem 2.3. Suppose that the data of Problem (2.1) satisfy the bound (2.2) and the smoothness conditions (2.4) and (2.7) with $\ell \geq 5$ and $M_{2}=M_{3}$. Then, for $\varepsilon \in(0,1]$, the bounds (2.11) and (2.17), with $0 \leq|\alpha| \leq 3$, hold for the regular and singular components $U, V$ of the solution $u$ defined in (2.6).

3. Nonlinear finite difference methods. Using the results of Theorem 2.2 finite difference methods are now constructed for Problem (2.1). On $\bar{\Omega}$ the mesh

$$
\bar{\Omega}_{N}=\bar{\omega}_{1} \times \omega_{2} \times \ldots \times \omega_{n}
$$

is introduced, where $\bar{\omega}_{1}$ is some mesh on the interval $[0, d]$ of the $x_{1}$-axis and $\omega_{r}$ is a uniform mesh on the $x_{r}$-axis with step size $h_{r}, r=2, \ldots, n$. Define $h_{1}^{i}=x_{1}^{i}-x_{1}^{i-1}, x_{1}^{i-1}, x_{1}^{i} \in$ $\bar{\omega}_{1}, h_{1}=\max _{i} h_{1}^{i}, h=\max _{r} h_{r}, r=1, \ldots, n$. Let $N_{1}$ denote the number of nodes in the mesh $\omega_{1}, N_{r}=1 / h_{r}, r=2, \ldots, n, N=\min _{r} N_{r}, r=1, \ldots, n$, and $M=N h<\infty$. It is assumed hereafter that

$$
a_{1, r}(x) \equiv 0, x \in \bar{\Omega}, r=2, \ldots, n .
$$

Hence no mixed finite differences involving partial differences in the $x_{1}$ direction occur.

On the uniform meshes $\omega_{r}, r=2, \ldots, n$, the following first order finite differences are defined

$$
\begin{aligned}
& D_{r}^{+} z(x)=\left(z\left(x+\epsilon_{r} h_{r}\right)-z(x)\right) / h_{r}, \\
& D_{r}^{-} z(x)=\left(z(x)-z\left(x-\epsilon_{r} h_{r}\right)\right) / h_{r},
\end{aligned}
$$

where $e_{r}$ is the unit vector along the $x_{r}$-axis. The following second order finite differences are also required on these meshes 


$$
\begin{aligned}
\delta_{r}^{2} z(x) & =\left(D_{r}^{+}-D_{r}^{-}\right) z(x) / h_{r}, \\
D_{r}^{+} D_{s}^{+} z(x) & =D_{r}^{+}\left(D_{s}^{+} z(x)\right), \\
D_{r}^{+} D_{s}^{-} z(x) & =D_{r}^{+}\left(D_{s}^{-} z(x)\right), \\
D_{r}^{-} D_{s}^{+} z(x) & =D_{r}^{-}\left(D_{s}^{+} z(x)\right), \\
D_{r}^{-} D_{s}^{-} z(x) & =D_{r}^{-}\left(D_{s}^{-} z(x)\right) .
\end{aligned}
$$

On the non-uniform mesh $\omega_{1}$ first and second order finite differences, for each $x=x^{i}=$ $\left(x_{1}^{i}, x_{2}, \ldots, x_{n}\right) \in \Omega_{N}$, are defined by

$$
\begin{aligned}
D_{1}^{+} z(x) & =\left(z\left(x^{i+1}\right)-z\left(x^{i}\right)\right) / h_{1}^{i} \\
D_{1}^{-} z(x) & =\left(z\left(x^{i}\right)-z\left(x^{i-1}\right)\right) / h_{1}^{i-1} \\
\delta_{1}^{2} z(x) & =2\left(D_{1}^{+}-D_{1}^{-}\right) z(x) /\left(h_{1}^{i}+h_{1}^{i-1}\right)
\end{aligned}
$$

The finite difference method for Problem 2.1, on the mesh $\bar{\Omega}_{N}$, is then defined by

$$
L^{h} u_{N}(x)=0, x \in \Omega_{N}, u_{N}(x)=\phi(x), x \in \partial \Omega_{N}
$$

where $\Omega_{N}=\Omega \cap \bar{\Omega}_{N}$ and

$$
L^{h} z(x) \equiv\left\{\varepsilon^{2} L_{2}^{h}+\varepsilon L_{1}^{h}\right\} z(x)-g(x, z(x))
$$

Here

$$
\begin{gathered}
L_{2}^{h} \equiv \sum_{r=1}^{n} a_{r r}(x) \delta_{r}^{2}+\sum_{r, s=2, r \neq s}^{n} 2^{-1}\left[a_{r s}^{+}(x)\left(D_{r}^{+} D_{s}^{+}+D_{r}^{-} D_{s}^{-}\right)\right. \\
\left.+a_{r s}^{-}(x)\left(D_{r}^{+} D_{s}^{-}+D_{r}^{-} D_{s}^{+}\right)\right]+\sum_{r=1}^{n}\left[b_{r}^{+}(x) D_{r}^{+}+b_{r}^{-} D_{r}^{-}\right]-c(x) \\
L_{1}^{h} \equiv \sum_{r=1}^{n}\left[b_{r}^{1+}(x) D_{r}^{+}+b_{r}^{1-}(x) D_{r}^{-}\right]-c^{1}(x)
\end{gathered}
$$

where $v^{+}(x)=(v(x)+|v(x)|) / 2$ and $v^{-}(x)=(v(x)-|v(x)|) / 2$.

By analogy with $\mathcal{L}$, we define the formal notation 


$$
\mathcal{L}^{h}(f(x, w)) z(x)=\left(\varepsilon^{2} L_{2}^{h}+\varepsilon L_{1}^{h}\right) z(x)-f(x, w(x)) .
$$

and it is clear that

$$
L^{h} z(x)=\mathcal{L}^{h}(g(x, z)) z(x) .
$$

Under the assumptions (3.2) and the additional assumption

$$
\frac{\left|a_{r s}(x)\right|}{h_{r} h_{s}}<\frac{1}{n-1} \min \left\{\frac{a_{r r}(x)}{h_{r}^{2}}, \frac{a_{s s}(x)}{h_{s}^{2}}\right\}, x \in \bar{\Omega}, r, s=1, \ldots, n, r \neq s
$$

the operator $L_{2}^{h}$ is monotone on $\Omega_{N}$ for any distribution of nodes in $\bar{\omega}_{1}$.

The finite difference method (3.3) is now examined. The error $w(x)=u_{N}(x)-u(x), x \in$ $\bar{\Omega}_{N}$, satisfies

$$
\mathcal{L}^{h}\left(\hat{g}_{u}\left(x, u_{N}, u\right)\right) w(x)=F_{1}^{h}(x, u), x \in \Omega_{N}, w(x)=0, x \in \partial \Omega_{N}
$$

where

$$
F_{1}^{h}(x, u) \equiv\left\{\varepsilon^{2}\left(L_{2}^{h}-L_{2}\right)+\varepsilon\left(L_{1}^{h}-L_{1}\right)\right\} u(x)
$$

and $\hat{g}$ is as in (2.9). The operator $\mathcal{L}^{h}\left(\hat{g}_{u}\left(x, u_{N}, u\right)\right)$ is monotone on $\bar{\Omega}_{N}$, for any distribution of nodes in $\bar{\omega}_{1}$, provided that conditions (3.2) and (3.5) are fulfilled.

However, these conditions are not sufficient to guarantee that the finite difference method (3.3) is $\varepsilon$-uniform. To obtain an $\varepsilon$-uniform numerical method a special mesh $\bar{\Omega}_{N}^{\star}$, which is refined or condensed in the boundary layer, is required. A piecewise uniform mesh having this property is now constructed. For $r=2, \ldots, n$ the uniform mesh $\omega_{r}$ is used, as before. In the interval $[0, d]$ of the $x_{1}$-axis a special mesh $\bar{\omega}_{1}^{\star}$ is defined as follows. The interval $[0, d]$ is partitioned into three subintervals $[0, \sigma],[\sigma, d-\sigma],[d-\sigma, d]$ where $\sigma \equiv \min \left[\frac{1}{4} d, M \varepsilon \ln N_{1}\right], M>\left[a^{0}\left(g_{0}\right)^{-1}\right]^{\frac{1}{2}}$. A uniform mesh is constructed using $N_{1} / 4$ points in each of $[0, \sigma],[d-\sigma, d]$ and $N_{1} / 2$ points in $[\sigma, d-\sigma]$.

Using the technique in [23] it is possible to prove that the finite difference operator $L^{h}$ in (3.3) on the mesh $\bar{\Omega}_{N}^{\star}$ gives an $\varepsilon$-uniform method. In particular the following $\varepsilon$-uniform error estimate holds

$$
\left|u(x)-u_{N}(x)\right| \leq M N^{-1} \ln N, x \in \bar{\Omega}_{N}^{\star} .
$$

The above results are summarized in the following theorem :

Th ео ем 3.1. Suppose that the data of Problem 2.4 satisfy the conditions (2.2), (2.4) and (2.7) and the assumptions (3.2) and (3.5). Suppose, also, that with $\varepsilon \in(0,1]$ and $0 \leq|\alpha| \leq 3$ the bound (2.5) holds for the solution $u$ and its derivatives and that the bounds (2.11), (2.17) hold for its regular and singular components $U, V$ respectively. Then, the numerical method comprising the nonlinear finite difference operator $L^{h}$ in (3.3) on the mesh $\bar{\Omega}_{N}^{\star}$ is $\varepsilon$-uniform and the $\varepsilon$-uniform error estimate (3.7) holds.

This theorem shows that in principle the problem is solved. In practice, however we are still faced with the problem of solving a nonlinear system of algebraic equations. This is dealt with in the next section. 
4. Iterative finite difference methods. Throughout this section it is assumed that (3.2) is satisfied. Analogously to $\mathcal{L}^{h}$ we define

$$
\mathcal{L}_{t}^{h}(f(x, w), p) z(x, t) \equiv\left(\varepsilon^{2} L_{2}^{h}+\varepsilon L_{1}^{h}-p D_{t}^{+}\right) z(x, t)-f(x, w(x, t)) .
$$

where the positive parameter $t$, which plays the part of a continuation parameter, will be determined later, and $D_{t}^{+}$is defined by

$$
D_{t}^{+} z\left(x, t_{j}\right)=\left(t_{j}-t_{j-1}\right)^{-1}\left(z\left(x, t_{j}\right)-z\left(x, t_{j-1}\right)\right) .
$$

We now consider the iterative finite difference method

$$
\mathcal{L}_{t}^{h}\left(g\left(x, u_{0}\right), p\right) u_{N}(x, t)=0,(x, t) \in G_{N}^{*}, u_{N}(x, t)=\phi(x, t),(x, t) \in \partial G_{N}^{*},
$$

where $u_{o}(x, t)$ is the old value of $u_{N}$ defined by $u_{o}\left(x, t_{j}\right)=u_{N}\left(x, t_{j-1}\right)$, and where the mesh $G_{N}^{*}$ is defined by

$$
\bar{G}_{N}^{*}=\bar{\Omega}_{N}^{*} \times \bar{\omega}_{t}
$$

with $\bar{\omega}_{t}$ being some mesh having $K+1$ points in the interval $[0, T]$, with $t_{0}=0$ and $t_{K}=T$. The boundary of this mesh is defined by

$$
\partial G_{N}^{*}=\partial G_{0, N}^{*} \cup \partial G_{d, N}^{*} \cup \partial G_{N}^{*, 0}
$$

where

$$
\partial G_{N}^{*, 0}=\left\{(x, t) \in \bar{G}_{N}^{*}, t=0\right\}, \quad \partial G_{0, N}^{*}=\left\{(x, t) \in \bar{G}_{N}^{*}, x_{1}=0\right\}
$$

and

$$
\partial G_{d, N}^{\star}=\left\{(x, t) \in \bar{G}_{N}^{*}, x_{1}=d\right\}
$$

The following boundary and initial conditions are imposed

$$
\phi(x, t)=\phi(x),(x, t) \in \partial G_{0, N}^{*} \cup \partial G_{d, N}^{*}, \quad \phi(x, t)=\phi^{0}(x),(x, t) \in \partial G_{N}^{*, 0},
$$

where $\phi^{0}(x)$ is a bounded function and, in general, $\phi \neq \phi^{0}$.

The finite difference method (4.1) is implicit. At each time level, the function $u_{N}(x, t)$ is the solution of a discrete linear boundary value problem. Note that the operator $\varepsilon^{2} L_{2}^{h}+$ $\varepsilon L_{1}^{h}-p D_{t}^{+}$is monotone on the mesh $G_{N}^{*}$ for any distribution of the mesh points in $\bar{\omega}_{t}$.

To investigate the convergence of this method, note that the error function $w(x, t)=$ $u_{N}(x, t)-u(x),(x, t) \in \bar{G}_{N}^{*}$ is the solution of the discrete problem

$$
\begin{gathered}
\mathcal{L}_{t}^{h}\left(\hat{g}_{u}\left(x, u_{0}, u\right), P\left(x, t, u_{o}(x, t), u(x)\right) w(x, t)=F_{1}^{h}(x, u),(x, t) \in G_{N}^{*},\right. \\
w(x, t)=\phi(x, t)-u(x)=\phi^{0}(x)-u(x),(x, t) \in \partial G_{N}^{*, 0},
\end{gathered}
$$




$$
w(x, t)=0,(x, t) \in \partial G_{0, N}^{*} \cup \partial G_{d, N}^{*},
$$

where $F_{1}^{h}$ is as in (3.6), $P\left(x, t, v_{1}, v_{2}\right)=p-\tau_{j} \hat{g}_{u}\left(x, v_{1}, v_{2}\right)$, and $\tau_{j}=t_{j}-t_{j-1}$. The operator $\mathcal{L}_{t}^{h}\left(\hat{g}_{u}\left(x, u_{0}, u\right), P\left(x, t, u_{o}(x, t), u(x)\right)\right.$ is monotone if

$$
\min _{\bar{G}_{N}^{*}} P\left(x, t, u_{o}(x, t), u(x)\right) \geq 0 .
$$

The error $w(x, t)$ is estimated using a discrete maximum principle (see for example [23]). This yields the discrete analogue of (2.3)

$$
\left|u_{N}(x, t)\right| \leq \max \left[g_{0}^{-1} \max _{\bar{\Omega}^{*}}|g(x, 0)|, \max _{\partial G_{N}^{*}}|\phi(x, t)|\right] .
$$

From this inequality and $(2.3)$, we obtain

$$
\left|\hat{g}_{u}\left(x, u_{o}(x, t), u(x)\right)\right| \leq M,(x, t) \in \bar{G}_{N}^{*} .
$$

The mesh $\bar{\omega}_{t}$ is chosen to be uniform. Then $\tau_{j}=h_{t}$ for all $j$. The parameter $p$ is chosen sufficiently large or the time step $h_{t}$ is chosen sufficiently small so that

$$
P\left(x, t, u_{o}(x, t), u(x)\right)=p-h_{t} \hat{g}_{u}\left(x, u_{o}(x, t), u(x)\right) \geq p_{0}>0,(x, t) \in G_{N}^{*} .
$$

Using a maximum principle, the following error estimate is obtained from (4.3)

$$
|w(x, t)| \leq M\left(N^{-1} \ln N+e^{-m_{4} t}\right),(x, t) \in \bar{G}_{N}^{*},
$$

if the constant $m_{4}$ is sufficiently small to ensure that

$$
\begin{gathered}
{\left[\min _{\bar{G}_{N}^{*}} \hat{g}_{u}\left(x, u_{o}(x, t), u(x)\right)\right]-h_{t}^{-1}\left(\exp \left(m_{4} h_{t}\right)-1\right) \max _{\bar{G}_{N}^{*}} P\left(x, t, u_{o}(x, t), u(x)\right)} \\
\geq g_{0}-h_{t}^{-1}\left(\exp \left(m_{4} h_{t}\right)-1\right)\left(p+h_{t} M\right) \geq 0
\end{gathered}
$$

Thus we have

$$
\left|u(x)-u_{N}(x, t)\right| \leq M\left(N^{-1} \ln N+q^{t / h_{t}}\right),(x, t) \in G_{N}^{*},
$$

where $q=\exp \left(-m_{4} h_{t}\right)$ and hence

$$
\left|u(x)-u_{N}\left(x, T_{0}\right)\right| \leq M\left(N^{-1} \ln N\right), x \in \bar{\Omega}_{N}^{*},
$$

where

$$
T_{0} \geq m_{4}^{-1} \ln \left(N(\ln N)^{-1}\right) .
$$


The number of iterations required is

$$
K=T_{0} / h_{t}=\frac{\ln \left(N(\ln N)^{-1}\right)}{h_{t} m_{4}} \leq M \ln N
$$

The above results are summarized in the following theorem.

THEOREM 4.1. Assume that the estimates (2.11), (2.17) for $|\alpha| \leq 3$ are satisfied by the solution of problem (2.1). Assume also that the conditions (2.8), (3.2) and (3.5) are satisfied. Then the solutions of (4.1) converge E-uniformly to the solution of Problem 2.1. The error estimate (4.9) is valid. The number of iterations required is given in (4.10).

5. Numerical Results. In this section numerical results are given for the following one dimensional examples of the semilinear problem (2.1)

$$
\begin{gathered}
\varepsilon^{2} \frac{d^{2}}{d x^{2}} u(x)-g(x, u(x))=0, x \in(0,1), \\
u(0)=A \quad u(1)=B
\end{gathered}
$$

where either

$$
g(x, u)=u-u^{3}
$$

or

$$
g(x, u)=u-u^{2}
$$

Various choices of $A$ and $B$ in the boundary conditions are examined.

The central difference operator

$$
L^{h} u_{N} \equiv \varepsilon^{2} \delta_{x}^{2} u_{N}-g\left(x, u_{N}\right)=0, u_{N}(0)=u(0) u_{N}(1)=u(1)
$$

on a special piecewise-uniform mesh $\varpi_{1}^{*}$ is used. The mesh $\varpi_{1}^{*}$ is constructed by dividing the interval into three subintervals $[0, \sigma],[\sigma, 1-\sigma],[1-\sigma, 1]$ where

$$
\sigma \equiv \min \{0.25, \varepsilon \ln N\}
$$

A uniform mesh is used on each subinterval, taking $N / 4, N / 2$, and $N / 4$ points in the respective intervals.

The nonlinear finite difference method $\left\{L^{h}, \omega_{1}^{*}\right\}$ is linearized using the continuation method (with the parameter $p=1$ ) given in $\$ 4$. That is

$$
\begin{gathered}
L_{t}^{h} u_{N} \equiv \varepsilon^{2} \delta_{x}^{2} u_{N}\left(x, t_{j}\right)-g\left(x, u_{N}\left(x, t_{j-1}\right)\right)-D_{t}^{+} u_{N}\left(x, t_{j}\right)=0, j=1, \cdots K \\
u_{N}\left(0, t_{j}\right)=u(0), u_{N}\left(1, t_{j}\right)=u(1) \text { for all } j,
\end{gathered}
$$




$$
u_{N}(x, 0)=u_{\text {init }}(x)
$$

Various starting values $u_{\text {init }}(x)$ are chosen. The number of iterations $K$ and the choice of uniform time step $h_{t}=t_{j}-t_{j-1}$ are discussed below. With the definition

$$
e(j) \equiv \max _{1 \leq i \leq N}\left|u_{N}\left(x_{i}, t_{j}\right)-u_{N}\left(x_{i}, t_{j-1}\right)\right| / h_{t}, \text { for } j=1,2, \cdots, K,
$$

the time step $h_{t}$ is chosen sufficiently small so that

$$
e(j) \leq e(j-1), \text { for } 1<j \leq K
$$

and the number of iterations $K$ is chosen such that

$$
e(K) \leq \mathrm{TOL}
$$

where TOL is some prescribed small tolerance.

The numerical solution is obtained as follows:

Start with $h_{t}=0.0625$. If, at some value of $j,(5.5)$ is not satisfied then halve the time step until (5.5) is satisfied. Continue the iterations until either (5.6) is satisfied or until $K=90$. If (5.6) is not satisfied, then repeat the entire process starting with $h_{t}=0.03125$.

The resulting values of $u_{N}(x, K)$ are taken as approximations to the solution of the continuous problem (5.1).

The problem is solved on a sequence of meshes, with $N=8,16,32,64,128,256,512,1024$ and for $\varepsilon=2^{-n}, n=1,2, \cdots j_{r e d}$, where $j_{r e d}$ is chosen so that $\varepsilon$ is a value at which the rate of convergence stabilizes, which normally occurs when, to machine accuracy, we are solving the reduced problem.

The errors $\left|u_{N}\left(x_{i}, K\right)-u\left(x_{i}\right)\right|$ are approximated on each mesh for successive values of $\varepsilon$ by $e_{\varepsilon, N}(i)=\left|u_{N}\left(x_{i}, K\right)-u^{I}\left(x_{i}, K\right)\right|$, where $u^{I}(x, K)$ is defined by linear interpolation on each subinterval $\left[y_{j-1}, y_{j}\right]$ by

$$
u^{I}(x, K)=u^{*}\left(y_{j-1}, K\right)+\left(u^{*}\left(y_{j}, K\right)-u^{*}\left(y_{j-1}, K\right)\right) \frac{x-y_{j-1}}{y_{j}-y_{j-1}}, 1 \leq j \leq 1024
$$

where the nodal values $\left\{u^{*}\left(y_{j}, K\right)\right\}_{j=0}^{1024}$ are obtained from the solution of the finite difference method $\left\{L_{t}^{h}, \omega_{1}^{*} \times \omega_{t}\right\}$ with $N=1024$. For each $\varepsilon$ and each $N$ the maximum nodal error is approximated by

$$
E_{\varepsilon, N}=\max _{i} e_{\varepsilon, N}(i)
$$

For each $N$, the $\varepsilon$-uniform maximum nodal error is approximated by

$$
E_{N}=\max _{\varepsilon} E_{\varepsilon, N}
$$

In what follows all calculations were carried out in double-precision FORTRAN 77 on an Hewlett-Packard/Apollo 730. 
A numerical method for solving (5.1) is $\varepsilon$-uniform of order $p$ on the mesh $\Omega_{N}=\left\{x_{i}, i=\right.$ $0,1, \ldots, N\}$ if

$$
\sup _{0<\varepsilon \leq 1} \max _{\Omega_{N}}\left|u(x)-u_{N}(x, K)\right| \leq C N^{-p},
$$

where $u$ is the solution of (5.1), $u_{N}$ is the numerical approximation to $u, C$ and $p>0$ are independent of $\varepsilon$ and $N$. An approximation to $p$, the $\varepsilon$-uniform rate of convergence, was determined using a variation of the double mesh method described in [5]. This involves calculating the double mesh error

$$
D_{\varepsilon, N}=\max _{\Omega_{N}}\left|u_{N}\left(x_{i}, K\right)-u_{2 N}^{I}\left(x_{i}, K\right)\right|,
$$

which is the difference between the values of the solution on a mesh of $N$ points and the interpolated value for the solution, at the same point, on a mesh of $2 N$ points. For each value of $N$ the quantities

$$
D_{N}=\max _{\varepsilon} D_{\varepsilon, N}, \quad p_{N}=\log _{2}\left(\frac{D_{N}}{D_{2 N}}\right),
$$

are computed. The values of $p_{N}$ are the approximations to $p$.

Tables 5.1-5.6 and 5.8 present numerical results for centered differences on the special mesh $G_{n}^{*}$ for the problem (5.1),(5.2). Table 5.1 gives the errors $E_{\varepsilon, N}$ and $E_{N}$ for problem (5.1),(5.2) with boundary conditions $u(0)=1, u(1)=1$ and initial guess $u_{\text {init }}=0$. Tables 5.2-5.6 present summary results for problem (5.1),(5.2), for various boundary values and initial guesses. In all of these examples, it is clear that the central difference scheme on the special mesh $G_{N}^{*}$ yields an $\varepsilon$-uniform method. It should be noted that, in the case of the numerical examples presented here, $g(u, x)$ does not satisfy the condition (2.2), and thus the method may in practice be $\varepsilon$-uniformly convergent even in cases where this condition is not satisfied. Moreover, the computed rate of $\varepsilon$-uniform convergence is approximately 1 , independent of the choice of the initial guess and of the boundary conditions. This indicates that the theoretical result given in (4.10) may be a conservative estimate of the rate of $\varepsilon$ uniform convergence. To verify this further we give in Table 5.7 below the local theoretical rates of $\varepsilon$-uniform convergence, corresponding to those in Tables 5.2-5.6. It is clear that the actual computed rates in Tables 5.2-5.6 are substantially better, in all cases than the theoretical rates.

We should also remark that, as shown in [7, pp. 124-127], there are multiple solutions to this problem for the boundary conditions, $u(0)=1, u(1)=1$. The stable solution has boundary layers at both end-points and, for sufficiently small $\varepsilon$, approaches the reduced solution $u(x) \equiv 0$ in the interior. On the other hand, it is clear that $u(x) \equiv 1$ is also a solution, in this case an unstable one. We remark that for all the initial guesses discussed above, the numerical solutions converge to the stable solution, and furthermore the computed rate of $\varepsilon$-uniform convergence obtained is the same. With the initial guess $u_{\text {init }}=1$ however, it converges to the unstable solution after one iteration as would be expected. On the other hand, it is of some interest, that for initial guesses as close to 1 as $u_{\text {init }}=.98$, the solutions converge to the stable solution, with essentially the same $\varepsilon$-uniform rate as the above examples. The results for this case are given in Table 5.8. 


\begin{tabular}{|c|c|c|c|c|c|c|c|}
\hline \multicolumn{8}{|c|}{$\begin{array}{rc}\text { Boundary Conditions: } & u(0)=1, u(1)=1 \\
\text { Initial Guess : } & u_{\text {init }}=0\end{array}$} \\
\hline \multirow[b]{2}{*}{$\varepsilon$} & \multicolumn{7}{|c|}{ Number of Mesh Points $N$} \\
\hline & 8 & 16 & 32 & 64 & 128 & 256 & 512 \\
\hline $1 / 2$ & .000055 & .000013 & .000003 & .000001 & .000000 & .000000 & .000000 \\
\hline $1 / 4$ & .000037 & .000634 & .000057 & .000014 & .000003 & .000001 & .000000 \\
\hline $1 / 8$ & .006885 & .001755 & .000440 & .000110 & .000027 & .000006 & .000001 \\
\hline $1 / 16$ & .006907 & .002070 & .000444 & .000111 & .000027 & .000007 & .000001 \\
\hline $1 / 32$ & .011231 & .003309 & .000878 & .000219 & .000054 & .000013 & .000003 \\
\hline 1/ 64 & .012372 & .006810 & .001777 & .000446 & .000111 & .000026 & .000005 \\
\hline $1 / 128$ & .016902 & .011381 & .003402 & .000899 & .000223 & .000053 & .000011 \\
\hline $1 / 256$ & .033105 & .011972 & .005121 & .001780 & .000443 & .000106 & .000021 \\
\hline $1 / 512$ & .056008 & .012978 & .005182 & .001950 & .000673 & .000226 & .000043 \\
\hline $1 / 1024$ & .077146 & .021205 & .005260 & .001952 & .000677 & .000230 & .000081 \\
\hline $1 / 2048$ & .094695 & .031616 & .007446 & .001971 & .000679 & .000230 & .000081 \\
\hline $1 / 4096$ & .108414 & .041192 & .012061 & .002426 & .000682 & .000230 & .000081 \\
\hline $1 / 8192$ & .118767 & .049207 & .016901 & .004247 & .000736 & .000231 & .000081 \\
\hline $1 / 16384$ & .126412 & .055532 & .021361 & .006463 & .001373 & .000231 & .000081 \\
\hline 1/ 32768 & .131978 & .060343 & .025123 & .008769 & .002272 & .000409 & .000082 \\
\hline $1 / 65536$ & .135994 & .063915 & .028117 & .010900 & .003350 & .000728 & .000108 \\
\hline $1 / 131072$ & .138874 & .066527 & .030410 & .012707 & .004469 & .001170 & .000202 \\
\hline $1 / 262144$ & .140930 & .068418 & .032123 & .014153 & .005503 & .001694 & .000348 \\
\hline $1 / 524288$ & .142394 & .069775 & .033379 & .015264 & .006380 & .002232 & .000544 \\
\hline $1 / 1048576$ & .143433 & .070745 & .034289 & .016095 & .007080 & .002723 & .000767 \\
\hline $1 / 2097152$ & .144171 & .071436 & .034943 & .016704 & .007614 & .003131 & .000985 \\
\hline $1 / 4194304$ & .144693 & .071926 & .035410 & .017143 & .008010 & .003450 & .001173 \\
\hline $1 / 8388608$ & .145062 & .072274 & .035742 & .017458 & .008297 & .003688 & .001321 \\
\hline $1 / 16777216$ & .145323 & .072519 & .035977 & .017681 & .008503 & .003861 & .001433 \\
\hline $1 / 33554432$ & .145508 & .072693 & .036143 & .017840 & .008649 & .003985 & .001514 \\
\hline$E_{N}$ & .145508 & .072693 & .036143 & .017840 & .008649 & .003985 & .001514 \\
\hline
\end{tabular}

Errors $E_{\varepsilon, N}$ and $E_{N}$ for problem (5.1),(5.2)

\begin{tabular}{|l|r|r|r|r|r|r|}
\hline \multicolumn{7}{|c|}{$\begin{array}{c}\text { Boundary Conditions: } \\
\text { Initial Guess : }\end{array}(0)=1, u(1)=1$} \\
$u_{\text {init }}=0$ \\
\hline \hline$N$ & 8 & 16 & 32 & 64 & 128 & 256 \\
\hline$E_{N}$ & .145508 & .072693 & .036143 & .017840 & 0.08649 & .003985 \\
\hline$p_{N}$ & 0.94 & 1.02 & 1.02 & 1.03 & 1.04 & 1.09 \\
\hline
\end{tabular}

Maximum errors $E_{N}$ and computed rates of convergence $p_{N}$

The final tables 5.9-13 give the corresponding numerical results for the problem (5.1),(5.3). Again, it may be observed that for all the initial guesses discussed, convergence to the true 


\begin{tabular}{|l|r|r|r|r|r|r|}
\hline \multicolumn{7}{|c|}{$\begin{array}{c}\text { Boundary Conditions: } u(0)=1, u(1)=1 \\
\text { Initial Guess : }\end{array} u_{\text {init }}=.5$} \\
\hline \hline$N$ & 8 & 16 & 32 & 64 & 128 & 256 \\
\hline$E_{N}$ & .145674 & .072779 & .036186 & .017861 & .008660 & .003990 \\
\hline$p_{N}$ & .94 & 1.02 & 1.02 & 1.03 & 1.04 & 1.09 \\
\hline
\end{tabular}

Maximum errors $E_{N}$ and computed rates of convergence $p_{N}$

\begin{tabular}{|l|r|r|r|r|r|r|}
\hline \multicolumn{7}{|c|}{$\begin{array}{r}\text { Boundary Conditions: } \\
\text { Initial Guess : }\end{array}(0)=.5, u(1)=.7$} \\
\hline init $=0$ \\
\hline \hline$N$ & 8 & 16 & 32 & 64 & 128 & 256 \\
\hline$E_{N}$ & .093222 & .046517 & .023123 & .011413 & .005533 & .002549 \\
\hline$p_{N}$ & .97 & 1.03 & 1.02 & 1.03 & 1.04 & 1.09 \\
\hline
\end{tabular}

Maximum errors $E_{N}$ and computed rates of convergence $p_{N}$

\begin{tabular}{|l|r|r|r|r|r|r|}
\hline \multicolumn{7}{|c|}{$\begin{array}{c}\text { Boundary Conditions: } \\
\text { Initial Guess : }\end{array}(0)=.5, u(1)=.7$} \\
$u_{\text {init }}=.5$ \\
\hline \hline$N$ & 8 & 16 & 32 & 64 & 128 & 256 \\
\hline$E_{N}$ & .093268 & .046541 & .023135 & .011419 & .005536 & .002551 \\
\hline$p_{N}$ & .97 & 1.03 & 1.02 & 1.03 & 1.04 & 1.09 \\
\hline
\end{tabular}

Maximum errors $E_{N}$ and computed rates of convergence $p_{N}$

\begin{tabular}{|l|r|r|r|r|r|r|}
\hline \multicolumn{7}{|c|}{ Boundary Conditions: $u(0)=.5, u(1)=.7$} \\
Initial Guess : & \multicolumn{3}{c|}{$u_{\text {init }}=u(0)+(u(1)-u(0)) x$} \\
\hline \hline$N$ & 8 & 16 & 32 & 64 & 128 & 256 \\
\hline$E_{N}$ & .093257 & .046535 & .023132 & .011417 & .005536 & .002550 \\
\hline$p_{N}$ & .97 & 1.03 & 1.02 & 1.03 & 1.04 & 1.09 \\
\hline
\end{tabular}

Maximum errors $E_{N}$ and computed rates of convergence $p_{N}$

\begin{tabular}{|l|r|r|r|r|r|r|}
\hline$N$ & 8 & 16 & 32 & 64 & 128 & 256 \\
\hline$p_{N}$ & .4150 & .5850 & .6781 & .7370 & 7776 & .8074 \\
\hline
\end{tabular}

Theoretical rates of convergence $p_{N}$ from (4.10)

solution occurs and the computed rate of $\varepsilon$-uniform convergence of the scheme is essentially the same, independent of the initial guess. 


\begin{tabular}{|l|r|r|r|r|r|r|}
\hline \multicolumn{7}{|c|}{ Boundary Conditions: $u(0)=1, u(1)=1$} \\
\hline \hline$N$ & 8 & 16 & 32 & 64 & 128 & 256 \\
\hline$E_{N}$ & .145661 & .072771 & .036182 & .017859 & .008659 & .003989 \\
\hline$p_{N}$ & .94 & 1.02 & 1.02 & 1.03 & 1.04 & 1.09 \\
\hline
\end{tabular}

Maximum errors $E_{N}$ and computed rates of convergence $p_{N}$

\begin{tabular}{|l|r|r|r|r|r|r|}
\hline \multicolumn{7}{|c|}{$\begin{array}{c}\text { Boundary Conditions: } \\
\text { Initial Guess : }\end{array}(0)=1, u(1)=1$} \\
$u_{\text {init }}=0$ \\
\hline \hline$N$ & 8 & 16 & 32 & 64 & 128 & 256 \\
\hline$E_{N}$ & .097937 & .048050 & .023691 & .011648 & .005637 & .002595 \\
\hline$p_{N}$ & 1.06 & 1.08 & 1.04 & 1.04 & 1.05 & 1.09 \\
\hline
\end{tabular}

Maximum errors $E_{N}$ and computed rates of convergence $p_{N}$

\begin{tabular}{|l|r|r|r|r|r|r|}
\hline \multicolumn{7}{|c|}{ Boundary Conditions: $u(0)=1, u(1)=1$} \\
\hline$N$ & 8 & 16 & 32 & 64 & 128 & 256 \\
\hline \hline$E_{N}$ & .097873 & .048014 & .023672 & .011638 & .005632 & .002593 \\
\hline$p_{N}$ & 1.06 & 1.08 & 1.04 & 1.04 & 1.05 & 1.09 \\
\hline
\end{tabular}

Maximum errors $E_{N}$ and computed rates of convergence $p_{N}$

\begin{tabular}{|l|r|r|r|r|r|r|}
\hline \multicolumn{7}{|c|}{$\begin{array}{r}\text { Boundary Conditions: } \\
\text { Initial Guess : }\end{array}(0)=.5, u(1)=.7$} \\
$u_{\text {init }}=0$ \\
\hline \hline$N$ & 8 & 16 & 32 & 64 & 128 & 256 \\
\hline$E_{N}$ & .073090 & .036008 & .017791 & .008755 & .004239 & .001952 \\
\hline$p_{N}$ & 1.05 & 1.07 & 1.04 & 1.03 & 1.05 & 1.09 \\
\hline
\end{tabular}

Maximum errors $E_{N}$ and computed rates of convergence $p_{N}$

\begin{tabular}{|l|r|r|r|r|r|r|}
\hline \multicolumn{7}{|c|}{$\begin{array}{c}\text { Boundary Conditions: } \\
\text { Initial Guess : }\end{array}(0)=.5, u(1)=.7$} \\
\hline init $=.5$ \\
\hline \hline$N$ & 8 & 16 & 32 & 64 & 128 & 256 \\
\hline$E_{N}$ & .073034 & .035977 & .017774 & .008747 & .004235 & .001950 \\
\hline$p_{N}$ & 1.05 & 1.07 & 1.04 & 1.03 & 1.05 & 1.09 \\
\hline
\end{tabular}

Maximum errors $E_{N}$ and computed rates of convergence $p_{N}$ 


\begin{tabular}{|l|r|r|r|r|r|r|}
\hline \multicolumn{6}{|c|}{ Boundary Conditions: $u(0)=.5, u(1)=.7$} \\
Initial Guess : & \multicolumn{4}{c|}{$u_{\text {init }}=u(0)+(u(1)-u(0)) x$} \\
\hline \hline$N$ & 8 & 16 & 32 & 64 & 128 & 256 \\
\hline$E_{N}$ & .073063 & .035993 & .017782 & .008751 & .004237 & .001951 \\
\hline$p_{N}$ & 1.05 & 1.07 & 1.04 & 1.03 & 1.05 & 1.09 \\
\hline
\end{tabular}

Maximum errors $E_{N}$ and computed rates of convergence $p_{N}$

[1] A. Brandt, I. Yavneh, Inadequacy of First-order Upwind Difference Schemes for some Recirculating Flows, J. Compuut. Phys., 93, (1991) pp. 128-143.

[2] E.P. Doolan, J.J.H. Miller, W.H.A. Schilders, Uniform Numerical Methods for Problems with Initial and Boundary Layers, Boole Press, Dublin, Ireland, 1980.

[3] T.M. El-Mistikawy, M.J. Werle, Numerical Method for Boundary Layers with Blowing - the Exponential Box Scheme, AIAA J., 16 (1978), pp. 749-751.

[4] P.A. Farrell, E.C. Gartland, Jr., On the Scharfetter-Gummel Discretization for Drift-Diffusion Continuity Equations, in "Computational Methods for Boundary and Interior Layers in Several Dimensions", J.J.H. Miller, ed., pp. 51-79, Boole Press, Dublin, Ireland, (1991).

[5] P.A. Farrell, A. Hegarty, On the determination of the order of uniform convergence, in Proc. of $13^{\text {th }}$ IMACS World Congress, Dublin, Ireland, 1991, pp. 501-502.

[6] P.A. Farrell, J.J.H. Miller, E. O'Riordan, G.I. Shishkin, On the nonexistence of $\varepsilon$-uniform finite difference methods on uniform meshes for semilinear two-point boundary value problems, to appear.

[7] K.W. Chang, F.A. Howes, Nonlinear Singular Perturbation Phenomena: Theory and Application, Appl. Math. Sciences, 56, Springer-Verlag, 1984.

[8] A.M. Il'in, Difference scheme for a differential equation with a small parameter affecting the highest derivative, Mat. Zametki, 6 (1969), pp. 237-248.

[9] O.A. Ladyzenskaya, N.N. Uralceva, Linear and Quasilinear Equations of Elliptic Type, Moscow, Nauka, 1973.

[10] V.D. Liseikin, On the numerical solution of second order equations with a small parameter affecting the highest derivatives, Chisl. Metody Mechaniki Splosh. Sredy, Novosibirsk, 14, n. 3 (1983), pp. $98-108$.

[11] J.A. Mackenie, K.W. Morton, Finite Volume Solutions of Convection-Diffusion Test Problems, Oxford Univeristy Computing Lab., Tech. Rep. 90/01 (1990).

[12] P.A. Markowich, C.A. Ringhofer, S. Selberherr, M. Lentini, A singular perturbation approach for the analysis of the fundamental semiconductor equations, IEEE Trans. Electron Devices, 30, n. 9 (1983), pp. $1165-1180$.

[13] J.J.H. Miller, G.I. Shishkin, On the construction of uniformly convergent finite difference schemes for singularly perturbed problems for a quasilinear equation, in Computational Methods for Boundary and Interior Layers in Several Dimensions, J.J.H. Miller, ed. Boole Press, Dublin, Ireland, 1991, pp. 103-118,

[14] J.J.H. Miller, W. Song, A Tetrahedral Mixed Finite Element Method for the Stationary Semiconductor Continuity Equations SIAM J. Numer. Anal. , 31 n. 1 (1994), pp. 196-216.

[15] J.J.H. Miller ed., Applications of Advanced Computational Mathods for Boundary and Interior Layers, Boole Press, Dublin, Ireland, (1993)

[16] J.D. Murray, Lectures on Nonlinear Differential Equation Models in Biology, Clarendon Press, Oxford, 1977.

[17] W.V. van Roosbroeck, Theory of flows of electrons and holes in germanium and other semiconductors, Bell Syst. Tech. J., 29 (1950), pp. 560-607.

[18] D.L. Scharfetter, H.K. Gummel, Large-signal analysis of a silicon Read diode oscillator, IEEE Trans. Electron Devices, 16, n. 1 (1969), pp. 64-77.

[19] G.I. Shishkin, Approximation of solutions of singularly perturbed boundary value problems with corner boundary layer, J. Vychisl. Mat. i Mat. Fiz., 27, n. 9 (1987), pp. 1360-1372. (Comput. Maths. Math. Phys., 27, n. 5 (1987), pp. 54-63.) 
[20] G.I. Shishkin, Grid approximation of boundary value problems with regular boundary layer, Part 1, Part 2, Preprint INCA, 1990.

[21] G.I. Shishkin, Difference scheme for solving elliptic equations with small parameters affecting the derivatives, Banach Centre Publ., Warsaw, 3 (1978), pp. 89-92.

[22] G.I. Shishkin, Grid approximation of singularly perturbed boundary value problems with a regular boundary layer, Sov. J. Numer. Anal. Math. Modelling, 4, n. 5 (1989), pp. 397-417.

[23] G.I. Shishkin, Grid approximation of a singularly perturbed boundary-value problem for a quasi-linear elliptic equation in the completely degenerate case, J. Vychisl. Mat. i Mat. Fis., 31, n. 12 (1991), pp. 1808-1825. (Comput. Maths. Math. Phys., 31, n. 12 (1991), pp. 33-46.)

[24] R. Vulanović, P.A. Farrell, P.Lin, Numerical Solution of Non-linear Singular Perturbation Problems Modeling Chemical Reactions, in "Applications of Advanced Computational Methods for Boundary and Interior Layers", J.J.H. Miller, ed., Boole Press, Dublin, Ireland, pp. 192-213 (1993)

[25] V. W. Weekman, Jr., R. L. Gorring, Influence of volume change on gas-phase reactions in porous catalysts, J. Catalysis 4 (1965), 260-270. 